\title{
Der Beitrag Gottlieb Burckhardts (1836-1907) zur Psychochirurgie in medizinhistorischer und ethischer Sicht
}

Dominik Gross

\section{Summary}

Psychosurgery is defined as the practice of destroying or removing healthy brain tissue in order to change behaviour. Although the neurologist Egas Moniz (1874-1955) is occasionally said to be the founder of psychosurgery, the first psychosurgical operations were performed by Gottlieb Burckhardt, a Swiss psychiatrist who practised in the late $19^{\text {th }}$ century. In 1891 he reported the results of topectomies on six patients suffering from different psychiatric diseases. The aim of the present article is to illuminate the life of Burckhardt as well as his contribution to psychosurgery. Special attention is paid to the ethical aspects of his operative interventions.

First of all, we have to make allowance for the fact that the therapeutic chances in $19^{\text {th }}$-century psychiatry were quite poor. Therefore, Burckhardts topectomies might have been seen as a new and hopeful way of therapy. But by analysing Burckhardt's case reports, it becomes clear that he did not intend to cure his patients but only to ameliorate their disturbing behaviour and their non-compliance. Burckhardt himself tried to justify the immense risk of his operations by referring to the necessity of progress in medicine. Although Burckhardt spoke of promising improvements, his positive appraisal can hardly be maintained. For this and other reasons, his contemporary colleagues mainly declined his methods and reports.

Priv. Doz. Dr. med. dent. Dr. phil. Dominik Gross, M.A., Institut für Geschichte der Medizin der Universität Würzburg, Oberer Neubergweg 10a, D-97074 Würzburg 


\section{Zusammenfassung}

Der Begriff «Psychochirurgie» ist definiert als gezielter operativer Eingriff am morphologisch gesunden Gehirn zum Zweck der Beeinflussung der Psyche. Obwohl der Neurologe Egas Moniz (1874-1955) gelegentlich als Begründer der Psychochirurgie angeführt wird, war es der schweizerische Psychiater Gottlieb Burckhardt, der ausgangs des 19. Jahrhunderts die ersten psychochirurgischen Eingriffe vornahm. Im Jahre 1891 berichtete er über Topektomien bei sechs Patienten mit psychiatrischen Grunderkrankungen. Ziel des vorliegenden Beitrages ist es, den Lebenslauf Burckhardts, aber auch seinen Beitrag zur Psychochirurgie näher zu beleuchten. Ein besonderes Augenmerk gilt den ethischen Implikationen seiner Operationen.

Hierbei ist zunächst zu berücksichtigen, dass die therapeutischen Möglichkeiten in der Psychiatrie des vergangenen Jahrhunderts sehr begrenzt waren. Vor diesem Hintergrund könnte man dazu neigen, in Burckhardts Topektomien eine neue und hoffnungsvolle Behandlungsform zu sehen. Eine genaue Analyse seiner Fallbeschreibungen zeigt indessen, dass Burckhardt keine Heilung der Patienten, sondern lediglich eine Abschwächung der bestehenden Verhaltensauffälligkeiten und eine Verbesserung der Compliance anstrebte. Dabei versuchte er die erheblichen Risiken seiner operativen Eingriffe mit dem Hinweis auf die Notwendigkeit ständigen medizinischen Fortschritts zu rechtfertigen. Wenngleich Burckhardt von ersten, vielversprechenden therapeutischen Erfolgen sprach, ist jenes positive Fazit bei näherer Betrachtung kaum aufrechtzuerhalten. Nicht zuletzt aus diesem Grund wurden seine Methoden und Berichte von den zeitgenössischen Kollegen mehrheitlich ignoriert.

\section{Einleitung}

Während die «Psychochirurgie» als Therapieform spätestens seit der Mitte der Siebzigerjahre dank einer öffentlich und nicht immer sachlich geführten Diskussion $^{1}$ bis weit über die Grenzen des Fachs hinaus Bekanntheit erlangte, wird der Beitrag des schweizerischen Psychiaters und Anstaltsleiters

1 Vgl. etwa Der Spiegel, «Umschaltung ins Lammfromme», Nr. 33 (1975) 32-45; Der Spiegel, «Unklare Diagnose», Nr. 38 (1975) 158f. Siehe auch die populärwissenschaftliche, mit dem effekthaschenden Untertitel «Operative Umpolung des Verhaltens» versehene Darstellung von Egmont R. Koch: ders., Chirurgie der Seele, Stuttgart 1978. Zu der über weite Strecken von polemischen Stellungnahmen geprägten Debatte über die Psychochirurgie in der Bundesrepublik Deutschland vgl. ferner Reinhard Adler/Rolf Saupe, Psychochirurgie: zur Frage einer biologischen Therapie psychischer Störungen, Stuttgart 1979,208-220. Letztere bieten auch einen Überblick über Beiträge zur Psychochirurgie im westdeutschen Fernsehen: ebd., 214-218. 
Gottlieb Burckhardt (1836-1907) zur Entwicklung jenes Verfahrens bislang - von einzelnen Ausnahmen abgesehen ${ }^{2}$ - nur zögerlich zur Kenntnis genommen.

Dabei ist Burckhardt durchaus zu den bekannten Vertretern der Psychiatriegeschichte zu zählen: Ein Blick in August Hirschs Biographisches Lexikon der hervorragenden Ärzte von 1929 zeigt, dass dieser hier als «Docent für Nervenkrankheiten» und «Director der Irrenanstalt in Préfargier» ausgewiesen ist ${ }^{3}$. Auch Alma Kreuter bietet in ihrem 1996 erschienenen umfassenden biographischen Lexikon Deutschsprachige Neurologen und Psychiater eine konzise Biographie Burckhardts ${ }^{4}$. In keinem der erwähnten Lexika finden sich indessen Hinweise auf sein frühes Engagement in der Psychochirurgie.

Nicht wenige Autoren schreiben die Einführung der chirurgischen Psychotherapie dem Nobelpreisträger und Begründer der präfrontalen Leukotomie Egas Moniz (1874-1955) zu․ Moniz $^{6}$ selbst verwies in seinem retrospektiven

2 Vgl. die etwa folgenden Kurzbeiträge: C. Gelma/L. Singer/A. Dany, «Le psychiatre de Préfargier Burckhardt», Cahiers de la Psychiatrie 5 (1951) 135-144; Yves Joanette/Brigitte Stemmer/Gil Assal/Harry Whitaker, «From Theory to practice, The Unconventional Contribution of Gottlieb Burckhardt to Psychosurgery», Brain and Language 45 (1993) 572-587; Christian Müller, «Gottlieb Burckhardt, père de la topectomie», Revue médicale de la Suisse romande 78/11 (1958) 726-730. Der Beitrag von Christian Müller ist in leicht modifizierter Form auch in englischer und deutscher Sprache verfügbar: vgl. ders., «Gottlieb Burckhardt, The Father of Topectomy», American Journal of Psychiatry 117 (1960) 461-463, sowie ders., Vom Tollhaus zum Psychozentrum. Vignetten und Bausteine zur Psychiatriegeschichte in zeitlicher Abfolge, Hürtgenwald 1993, 143-146.

3 Biographisches Lexikon der hervorragenden Ärzte aller Zeiten und Völker, hg. von August Hirsch, Bd. 1, Berlin ${ }^{2} 1929,770$.

4 Alma Kreuter, Deutschsprachige Neurologen und Psychiater, München 1996,211f.

5 Vgl. etwa Henrique J. de Barahona Fernandez, «Egas Moniz», in: Kurt Kolle, Grosse Nervenärzte, Bd. 1, Stuttgart/New York 21970, 187-199; Der Spiegel, "Umschaltung ins Lammfromme», Nr. 33 (1975) 34 («Es war im Dezember 1935, am Tag nach Weihnachten, als der portugiesische Psychiater und Gehirnchirurg Egas Moniz zum ersten Male das Hirn eines Menschen operierte, um dessen Verhalten gezielt zu verändern.»); Jon Tippin/Fritz A. Henn, «Modified Leukotomy in the Treatment of Intractable Obsessional Neurosis», American Journal of Psychiatry 139 (1982) 1601-1603, insb. 1601 («Moniz was the first to show that a neurosurgical procedure could be effectively used in treating mental disorders.»); Thomas Röder/Volker Kubillus (Hgg.),Die Männer hinter Hitler. Wer die geheimen Drahtzieher hinter Hitler wirklich waren ... und unter welchem Deckmantel sie noch immer unter uns weilen, Malters 1994,300 («Bereits 1935, also drei Jahre vor dem Einsatz der Elektroschocktherapie, nutzen die Psychochirurgen ihre Skalpelle. Ein Portugiese war es, der erstmals diesen bemerkenswerten Eingriff vornahm.»)

Auch Code et al. weisen in ihren Beiträgen zur Neuropsychologie auf den geringen Bekanntheitsgrad von Burckhardts Operationen hin: Chris Code/Claus-W. Wallesch/Yves Joanette/André Roch Lecours (Hgg.), Classic Cases in Neuropsychology, Hove 1996, Werbetext zum Buch («The editors were concerned with the important single case. It may be ancient [Broca's case Le Borgne] or modern [Marshall \& Newcombe's deep dyslexic G. R.], famous [Phineas Gage] or less well-known [Burckhardt's early psychological cases]»).

6 Egas Moniz (1874-1955). Zum Werk Moniz' vgl. de Barahona Fernandez, a.O. (oben Anm. 2) 187-199. 
Beitrag «Mein Weg zur Leukotomie» zwar auf eine Reihe von richtungsweisenden Vorarbeiten, nahm jedoch mit keinem Wort auf die frühen Topektomien Burckhardts Bezug ${ }^{7}$. Auch von einzelnen deutschsprachigen Autoren wurde der Beitrag Burckhardts zur Psychochirurgie noch in den Siebzigerjahren als neue Erkenntnis ausgegeben ${ }^{8}$. Dies muss insofern überraschen, als Burckhardt seine Arbeit bereits 1890 in Berlin einer erstaunten Fachwelt vorstellte, und überdies 1891 in der bekannten deutschsprachigen Zeitschrift Allgemeine Zeitschrift für Psychiatrie und psychisch-gerichtliche Medizin einen 86seitigen (!) Artikel über seine psychochirurgischen Erfahrungen veröffentlichte ${ }^{9}$.

Andere Verfasser nehmen zwar - zumeist am Rande - auf Burckhardts frühe Operationen Bezug, irren jedoch hinsichtlich ihrer Datierung. Die diesbezüglichen Jahresangaben reichen von $1881^{10}$ über $1890^{11}$ und $1991^{12}$ bis zum Jahr $1895^{13}$. Tatsächlich gibt Burckhardt selbst an, seine ersten psychochirurgischen Eingriffe im Jahr 1888 durchgeführt zu haben ${ }^{14}$.

Ziel des vorliegenden Beitrages ist es, das Leben und Werk des schweizerischen Psychiaters näher zu beleuchten. Während der erste Teil des Aufsatzes der Biographie Burckhardts gewidmet ist, verengt sich der Blick im zweiten Kapitel auf seine psychochirurgischen Eingriffe. Über das operative Vorgehen hinaus interessieren hierbei Burckhardts Beweggründe und Motive, seine Indikationsstellungen, seine Erwartungen an die Therapie, aber

7 Vgl. Egas Moniz, «Mein Weg zur Leukotomie», Deutsche Medizinische Wochenschrift 73 (1948) 581-583.

$8 \mathrm{Vgl}$. etwa die ansonsten sehr kenntnisreiche Monographie von Adler/Saupe zur Psychochirurgie: a. O. (wie Anm. 1) 38 («Merkwürdigerweise war die Tatsache, dass Burckhardt schon 1888 die ersten psychochirurgischen Eingriffe durchführte, bis vor kurzem kaum bekannt.»). Als Gegenbeispiel kann Heinrich Schipperges herangezogen werden. Letzterer nahm bereits in den Fünfzigerjahren - wenngleich am Rande - auf die Versuche Burckhardts Bezug: ders., «Zur Entwicklung der Psychochirurgie», Ciba-Zeitschrift 75 (1955) 2491-2497, hier 2495.

9 Gottlieb Burckhardt, «Ueber Rindenexcisionen, als Beitrag zur operativen Therapie der Psychosen», Allgemeine Zeitschrift für Psychiatrie und psychisch-gerichtliche Medizin 47 (1891) 463-548.

10 Oscar Sugar, «Changing Attitudes toward Psychosurgery», Surgical Neurology 9 (1978) 331-335, hier 331.

11 Vgl. Erwin Ackerknecht, Kurze Geschichte der Psychiatrie, Stuttgart ${ }^{2} 1967,103$. Ackerknecht nimmt in seinem psychiatriegeschichtlichen Abriss nur mit einem Satz auf Burckhardt Bezug: ebenda.

12 Koch, a. O. (oben Anm. 1) 15; Elliot S. Valenstein, «The prefrontal area and psychosurgery», Progress in Brain Research 85 (1990) 539-554, hier 539.

13 Francis Schiller, «The Mystique of the Frontal Lobes», Gesnerus 42 (1985) 412-424, hier 424.

14 Burckhardt selbst gibt in seinem 1891 erschienenen Beitrag «Ueber Rindenexzisionen, als Beitrag zur operativen Therapie der Psychosen» expressis verbis den 29. Dezember 1888 als Datum der ersten Operation an: ders., a. O. (oben Anm. 9) 479. Auch im Jahresbericht 1888 der Heilanstalt Préfargier nennt Burckhardt dieses Datum: vgl. Joanette et al., a. O. (wie Anm. 2) 575. 
auch seine persönliche Bewertung der erzielten Ergebnisse. Im dritten und letzten Abschnitt der Arbeit wird der Versuch unternommen, die ethischen Implikationen der Burckhardtschen Eingriffe zu thematisieren. Zudem gilt es eine Erklärung zu finden für die Tatsache, dass eine kritische Auseinandersetzung mit dem Leben und Werk Burckhardts bis dato allenfalls ansatzweise erfolgt ist. Schliesslich interessiert die Frage, warum Gottlieb Burckhardt bis auf den heutigen Tag weitaus weniger mit der Psychochirurgie assoziiert wird als spätere Verfechter jener Therapieform wie etwa Egas Moniz und Walter Freeman ${ }^{15}$.

\section{Gottlieb Burckhardt (1836-1907)}

Burckhardt (Abb. 1) entstammte einer traditionsreichen Basler Ärztefamilie: Sowohl sein Grossvater Johann Rudolf Burckhardt als auch sein Vater August Burckhardt traten als Hochschullehrer an der Medizinischen Fakultät in Basel hervor ${ }^{16}$. Während der 1829 gestorbene Johann Rudolf Burckhardt eine Professur für "praktische Medizin» innehatte ${ }^{17}$, war August Burckhardt bis 1857 als Privatdozent für gerichtliche Medizin und Chirurgie tätig ${ }^{18}$.

Gottlieb Burckhardt wurde am 24. Dezember 1836 in Basel geboren ${ }^{19}$. Nach dem Besuch des städtischen Gymnasiums studierte er Medizin an den Universitäten Basel, Göttingen und Berlin. 1860 promovierte Burckhardt im Alter von knapp 24 Jahren zum Doctor medicinae. In seiner Dissertation beschäftigte er sich mit dem Epithel der ableitenden Harnwege ${ }^{20}$. Noch im gleichen Jahr trat Burckhardt der «Medizinischen Gesellschaft» in Basel bei ${ }^{21}$.

Schon 1862 habilitierte er sich an der Universität Basel für die Fachbereiche Innere Medizin und Nervenheilkunde. In den ersten Jahren seiner

15 Walter Freeman (1895-1972) führte zusammen mit James W. Watts die ersten Leukotomien in den USA durch (1936) und sorgte für eine rasche Verbreitung der Methode; vgl. auch dies., Psychosurgery, Springfield 1942.

16 Albert Teichmann (Hrsg.), Die Universität Basel in den 50 Jahren seit ihrer Reorganisation im Jahre 1835, Basel 1885, 51f.

17 Hagenbach, «[Nachruf] Dr. Gottlieb Burckhardt», Correspondenz-Blatt für Schweizer Ärzte 37 (1907) 257-260, hier 258; Teichmann, a. O. (oben Anm. 16) 51.

18 Teichmann a. O. (oben Anm. 16) 51.

19 Für die nachfolgenden biographischen Daten vgl. Biographisches Lexikon (oben Anm. 7) 770; Teichmann, a. O. (oben Anm. 13) 52; C. Bach, «Nekrolog Dr. G[ottlieb] Burckhardt», Allgemeine Zeitschrift für Psychiatrie und psychisch-gerichtliche Medizin 64 (1907) 529-534; [J. Bresler], «[Nachruf] Dr. Gottlieb Burckhardt», Psychiatrisch-neurologische Wochenschrift 8 (1907) 464; Hagenbach, a. O. (oben Anm. 17) 257-260.

20 Gottlieb Burckhardt, Ueber den Bau und das Verhalten des Epithelium der ableitenden Harnwege in normalen und pathologischen Zuständen, med. Diss. Basel 1860.

21 Hagenbach, a. O. (oben Anm. 17) 259. 


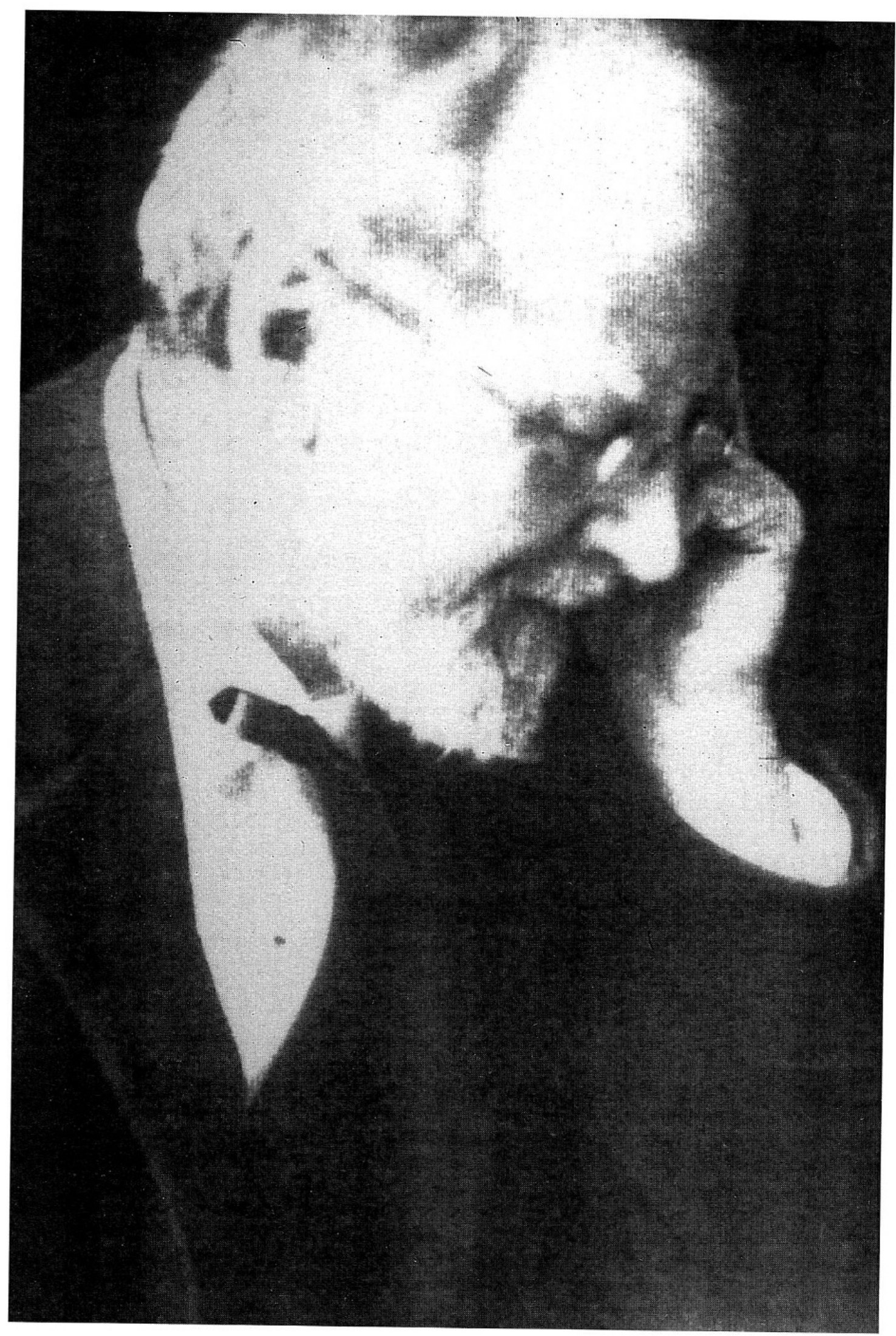

Abb. 1. Der schweizerische Psychiater und Neurochirurg Gottlieb Burckhardt (1836-1907). 
ärztlichen Tätigkeit führte er in seiner Heimatstadt eine Praxis. 1864 sah er sich jedoch aus gesundheitlichen Gründen gezwungen, seine praktische Arbeit vorübergehend aufzugeben ${ }^{22}$. Bis zu seiner Genesung verweilte er in dem südwestfranzösischen Kurort Pau ${ }^{23}$.

Nach seiner Rückkehr nach Basel wandte er sich verstärkt der Nervenheilkunde und hier insbesondere der aufkommenden Elektrotherapie $\mathbf{z u}^{24}$. 1873 wurde er gleichzeitig zum Präsidenten der Basler «Medizinischen Gesellschaft» und des Schweizerischen «Ärztlichen Centralvereins» gewählt ${ }^{25}$.

1875 legte er in Form der Monographie Die physiologische Diagnostik der Nervenkrankheiten einen zusammenfassenden Bericht über seine neurophysiologischen Untersuchungen vor $^{26}$.

Noch im selben Jahr nahm er eine Stelle als Sekundararzt an der psychiatrischen Anstalt Waldau bei Bern an $^{27}$. Gleichzeitig verliess er die «Medizinische Gesellschaft» in Basel, die ihm als Anerkennung für seine Leistungen die Ehrenmitgliedschaft verlieh ${ }^{28}$. Seit 1876 war Burckhardt neben seiner Tätigkeit in der Waldau an der Berner Hochschule als Privatdozent für Psychiatrie und Nervenkrankheiten tätig. Seine Probevorlesung war der Gehirnanatomie gewidmet. Sie wurde 1877 unter dem Titel «Die Lehre von den functionellen Centren des Gehirns und ihre Beziehung zur Psychologie und Psychiatrie» in der Allgemeinen Zeitschrift für Psychiatrie und psychisch-gerichtliche Medizin veröffentlicht ${ }^{29}$. Burckhardt blieb jenem Publikationsorgan in den nachfolgenden Jahren als Mitarbeiter eng verbunden. Allein in den Jahren 1881 bis 1895 war er hier mit insgesamt 16 Literaturberichten vertreten ${ }^{30}$.

22 Bach, a. O. (oben Anm. 19) 529.

23 Siehe Hagenbach, a. O. (oben Anm. 17) 258.

24 Vgl. etwa Gottlieb Burckhardt, «Ueber die polare Methode», Deutsches Archiv für klinische Medizin 8 (1871) 100-115.

25 Vgl. «Vereinsberichte», Correspondenz-Blatt für Schweizer Ärzte 3 (1873) 292-302 u. 547-552; Hagenbach, a. O. (oben Anm. 17) 259.

26 Vgl. Gottlieb Burckhardt, Die physiologische Diagnostik der Nervenkrankheiten. Versuch einer Feststellung der Leitungs- und Zuckungsverhältnisse im Nervensystem des gesunden und kranken Menschen, Leipzig 1875.

27 Während Bach und Hagenbach korrekterweise das Jahr 1875 anführen, datiert Müller die Publikation des Buches in das Jahr 1873: vgl. Bach, a. O. (oben Anm. 19) 529; Hagenbach, a. O. (oben Anm. 17) 258; Müller, Father of Topectomy (oben Anm. 2) 461.

28 Hagenbach, a. O. (oben Anm. 17) 260.

29 Gottlieb Burckhardt, «Die Lehre von den functionellen Centren des Gehirns und ihre Beziehung zur Psychologie und Psychiatrie», Allgemeine Zeitschrift für Psychiatrie und psychisch-gerichtliche Medizin 33 (1877) 434-454.

$30 \mathrm{Zu}$ den Literaturberichten von Gottlieb Burckhardt vgl. Allgemeine Zeitschrift für Psychiatrie und psychisch-gerichtliche Medizin 37 (1881) 443; 38 (1882) 27 u. 60; 40 (1884) 139; 41 (1885) 104*; 42 (1886) 280*; 43 (1887) 236*; 44 (1888) 257*; 45 (1889) 202*; 46 (1890) $141 * ;$ 47 (1891) 149*; 48 (1892) 164*; 49 (1893) 164* u. 369*; 50 (1894) 50, 224* u. 265; 51 (1895) 263*. (Das Symbol '*' kennzeichnet die Seiten des Anhangs der jeweiligen Zeitschriftenausgabe.) 
In der Waldau arbeitete Burckhardt unter Rudolph Schärer, der die Anstalt seit 1859 leitete ${ }^{31}$. Es ist davon auszugehen, dass Burckhardt mit seinem Vorgesetzten ein gutes Einvernehmen hatte. So verfasste er nach Schärers Ableben im Jahr 1890 nicht nur einen sehr wohlmeinenden Nachruf in der Allgemeinen Zeitschrift für Psychiatrie und psychisch-gerichtliche Medizin, sondern berichtete auch über regelmässige gemeinsame Freizeitaktivitäten mit seinem Vorgesetzten. Besonders an Samstagabenden versammelte Schärer Freunde und Mitarbeiter um sich, um Anekdoten auszutauschen oder zu musizieren ${ }^{32}$. Das musische Interesse dürfte Schärer und Burckhardt verbunden haben, denn letzterer war ein leidenschaftlicher Sänger, Violinist und Gitarrist ${ }^{33}$. Sein Talent nutzte Burckhardt sowohl zur eigenen Zerstreuung als auch zur Unterhaltung seiner Patienten ${ }^{34}$.

Im Zeitraum seiner Tätigkeit in Bern (1875-1882) veröffentlichte Burckhardt insgesamt 14 Fachbeiträge, so zum Beispiel eine Festschrift zu Ehren Albrecht von Hallers (1877) und mehrere Zeitschriftenaufsätze wie etwa die «Beobachtungen über die Temperaturen Geisteskranker» (1878) und ein Bericht über einen «Fall von Wortblindheit» $(1882)^{35}$.

31 Rudolph Schärer (1823-1890), Pfarrersohn, Studium der Medizin, seit 1849 Sekundararzt in Bern, seit 1855 Arzt in der Anstalt Waldau, seit 1859 Direktor ebenda: vgl. hierzu Gottlieb Burckhardt, «Nekrolog Schärer», Allgemeine Zeitschrift für Psychiatrie und psychisch-gerichtliche Medizin 47 (1891) 209-215, insb. 210.

32 Burckhardt, a. O. (oben Anm. 31) 210f.

33 Hagenbach, a. O. (oben Anm. 17) 259.

34 Vgl. Bach, a. O. (oben Anm. 19) 533f.

35 Nicht berücksichtigt sind hierbei die zahlreichen Literaturberichte (vgl. etwa Anm. 30). Für die erwähnten Schriften vgl. Anm. 26 und 29 sowie Gottlieb Burckhardt, «Nekrolog Professor Friedrich Brenner», Correspondenz-Blatt für Schweizer Ärzte 5 (1875) 65-69; ders., «Fall von idiopathischem Herzabscess», Correspondenz-Blatt für Schweizer Ärzte 6 (1876) 473-480; ders., «Ueber sensible und motorische Leitung im Rückenmark», Correspondenz-Blatt für Schweizer Ärzte 6 (1876) 544 u. 562; ders., Festschrift dem Andenken von Albrecht von Haller dargebracht von den Ärzten der Schweiz am 12. Dec. 1877, Bd. 2: Über Sehnenreflexe, Bern 1877, insb. 1-36; ders., «Beobachtungen über die Temperaturen Geisteskranker. Psychiatrische Beiträge», Archiv für Psychiatrie und Nervenkrankheiten 8 (1878) 333-354; ders., «Gemeine Rachsucht, unerlaubte Selbsthilfe oder Wahnsinn? Ein psychiatrisches Gutachten», Vierteljahresschrift für gerichtliche Medizin, n. F. 31 (1879) 235-251; ders., «Zweifelhafter Fall von Querulantenwahnsinn», Vierteljahresschrift für gerichtliche Medizin 31 (1879), Heft 2; ders., «Atrophie des Gyrus angularis nach Verlust eines Auges», in: Bericht pro 1879. Berner Canton-Anstalt Waldau [Bern 1880]; ders., «Ein Fall von Brandstiftung (epileptisches Irresein)», Correspondenz-Blatt für Schweizer Ärzte 11 (1881) 577-586; ders., «Ueber Gehirnbewegungen. Eine Experimentalstudie», in: Verhandlungen der Bernischen naturforschenden Gesellschaft, Bern 1881, 35-97; ders., «Die Mikrotomie des frischen Gehirns», Zentralblatt für die medizinischen Wissenschaften 29 (1881) 529; ders., «Ein Fall von Worttaubheit», Correspondenz-Blatt für Schweizer Ärzte 12 (1882) 673-682. In demselben Jahresband der Zeitschrift veröffentlichte Burckhardt zudem zwei Rezensionen. Sie betrafen eine Studie von B. Naunyn u. J. Schreiber über den «Gehirndruck» (S. 79f.) und eine Abhandlung von B. Frölich über die «Meningitis cerebrospinalis» (S. 345). 


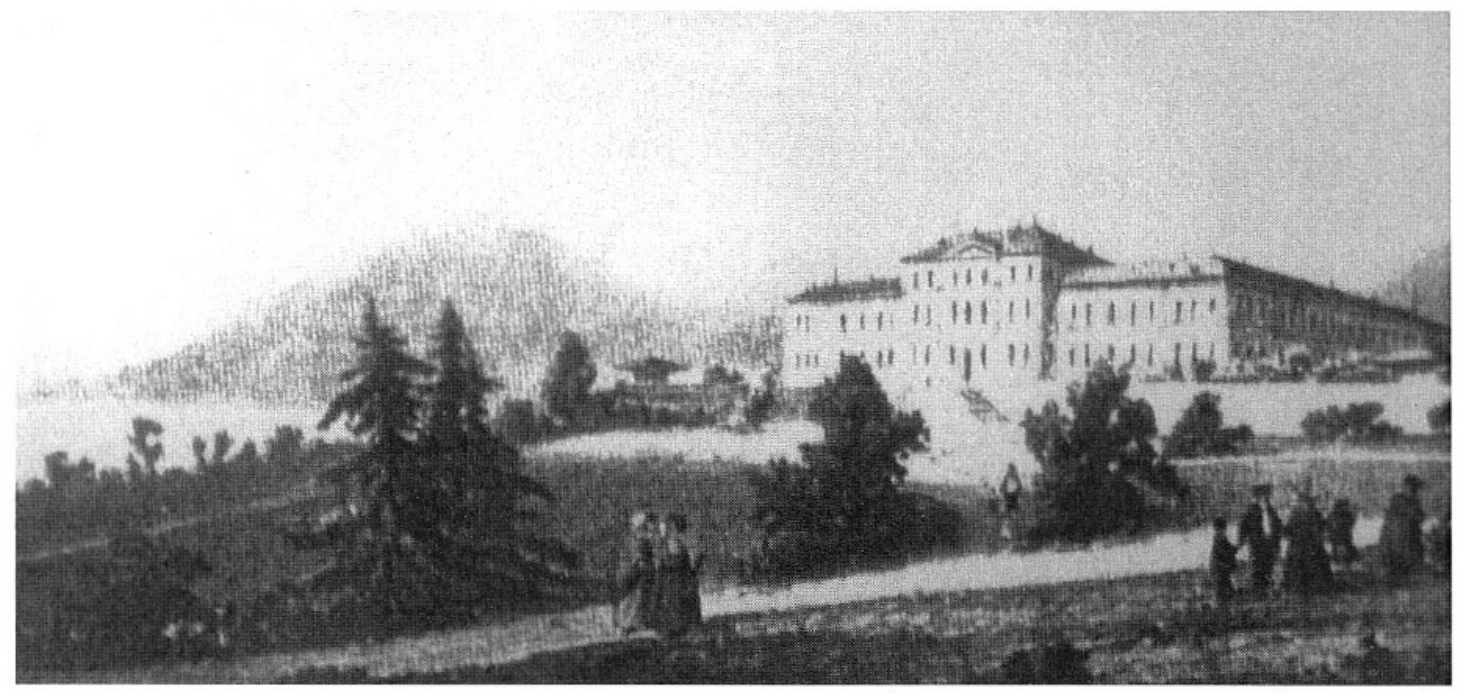

Abb. 2. Die Nervenheilanstalt in Préfargier am See von Neuchâtel um 1880.

Seit 1881 arbeitete Burckhardt u.a. mit dem jungen Paul Eugen Bleuler ${ }^{36}$ zusammen, der Jahre später wesentliche Beiträge zur Erforschung der Schizophrenie liefern sollte ${ }^{37}$.

Nach sechsjähriger Tätigkeit in Bern wurde Burckhardt 1882 die Leitung der Irrenanstalt in Préfargier bei Neuchâtel angetragen ${ }^{38}$. Im gleichen Jahr wurde sein jüngerer Bruder Albrecht Eduard Burckhardt von der Medizinischen Fakultät in Basel zum Privatdozenten für Toxikologie und Hygiene ernannt ${ }^{39}$.

Die Klinik in Préfargier war 1849 von dem wohlhabenden Industriellen Auguste-Frédéric de Meuron gegründet worden und zeichnete sich durch eine malerische Lage am Neuenburger See aus (vgl. Abb. 2). Gottlieb Burckhardt trat die Nachfolge von Dr. Auguste Châtelain an, der die Anstalt seit 1872 geleitet hatte, zuvor aber bereits als ärztlicher Mitarbeiter tätig gewesen war. Zwischen 1888 und 1890 führte Burckhardt hier an einigen Patienten die oben angesprochenen hirnchirurgischen Eingriffe durch ${ }^{40}$.

36 Paul Eugen Bleuler (1857-1939) führte das Konzept der Schizophrenie ein: vgl. ders., Dementia praecox oder die Gruppe der Schizophrenen, Leipzig/Wien 1911 - vgl. hierzu auch Jeremy M. Norman (Hrsg.), [Leslie Thomas] Morton's Medical Bibliography. An Annotated Check-List of Texts Illustrating the History of Medicine, London 51991,763; Kurt Kolle, Grosse Nervenärzte, Bd. 1, Stuttgart/New York ${ }^{2} 1970,7-16$.

37 Vgl. Bleuler, a. O. (oben Anm. 36).

38 Teichmann, a. O. (oben Anm. 16) 52.

39 Albrecht Eduard Burckhardt wurde am 13. Juli 1853 in Basel geboren; 1878 erfolgte seine Promotion zum Dr. med.: vgl. ebenda.

$40 \mathrm{Vgl}$. hierzu Teil 2 dieses Beitrags. 
Burckhardt verfügte laut Einschätzung seines Kollegen C. Bach von Natur aus über einen starken Willen und eine natürliche Autorität, die ihn zur Führungskraft geradezu prädestinierte: «Überhaupt war er ein geborener Anstaltsleiter, ein geborener Herrscher und wurde hierbei durch ein ausgezeichnetes Gedächtnis unterstützt ... Das, was die Franzosen 〈l'œil du maître〉 nannten, war bei ihm sehr entwickelt.» ${ }^{41}$

Sowohl die Patienten als auch das Klinikpersonal leisteten seinen Anordnungen Folge: «Nicht nur in der Hypnose, sondern auch in der Wachsuggestion und im alltäglichen Verkehr mit seinen Kranken hat er eine ungewöhnliche Macht auf ihre Gemüter ausgeübt. Sie mussten ihm gehorchen, ob sie wollten oder nicht, und auch das Wartepersonal fühlte sich unter seinem Banne. Besonders die willensschwachen Kranken rankten sich an ihm wie Epheu an einer Eiche empor. Denn er war eine markante, willensstarke Persönlichkeit.» ${ }^{42}$ Gleichzeitig würdigte Bach Burckhardts «diplomatische Gewandtheit», seine «unverbrüchliche Diskretion» und sein gutes Verhältnis zu den Patienten, denen er «als eine Art ärztlicher 〈Beichtvater»» gegenübergetreten $\mathrm{sei}^{43}$.

In therapeutischer Hinsicht legte Burckhardt «grosses Gewicht auf die medikamentöse Behandlung»; Bach zufolge glaubte er «an die objektive Wirkung der von ihm verordneten Arzneimittel» ${ }^{44}$. In diesem Punkt unterschied er sich deutlich von seinem früheren Vorgesetzten Rudolph Schärer, von dem Burckhardt selbst berichtete: «Der ärztliche Standpunkt Schärer's beabsichtigte mehr durch Verbesserung der Ernährung, durch eine richtig geleitete psychische und physische Diäthetik, als durch positive medicamentöse Massnahmen einzuwirken.» ${ }^{45}$ Auch hatte Schärer im Unterschied zu Burckhardt «keine Arbeiten weder anatomisch-experimenteller noch literarischkritischer Natur» verfasst ${ }^{46}$.

41 Bach, a. O. (oben Anm. 19) 532f.

42 Ebenda.

43 Ebenda.

44 Bach, a. O. (oben Anm. 19) 531.

45 Burckhardt, a. O. (oben Anm. 31) 212f.

46 Burckhardt, a. O. (oben Anm. 31) 213. Burckhardt setzte auch in Préfargier seine Publikationstätigkeit fort: vgl. ders., «Contribution à l'étude de l'hystérie traumatique», Revue médicale de la Suisse romande 6 (1886) 735-746; ders., «Hypnotismus», Correspondenz-Blatt für Schweizer Arzte 17 (1887) 562-564; ders., "Application de L'hypnotisme au traitement des maladies mentales», Revue de l'hypnotisme et de la psychologie physiologique 3 (1888/89) 56-59; ders., Maison de Santé de Préfargier, exercice de 1887.39me rapport annuel, Neuchâtel 1888; ders., "Un cas de tumeur de la couche optique et du lobe temporal», Revue médicale de la Suisse romande 8 (1888) 727-740; ders., «Weitere Mittheilungen über Gefässbewegungen. Theoretisches und Practisches», Archiv für Psychiatrie und Nervenkrankheiten 20 (1889) 605-627; ders., «Wiederholte Brandstiftung in Folge von moralischem Irresein», Friedreich's Blätter für gerichtliche Medizin und Sanitätspolizei 41 (1890) 270-278. Siehe auch ders., a. O. (oben Anm. 9) 463-548. 
Doch Burckhardt vertraute nicht allein der medikamentösen Therapie. Auch die Psychotherapie verstand er Bach zufolge «meisterhaft» ${ }^{47}$. Hypnose, Hydrotherapie und Elektrotherapie kamen unter dem Direktorat Burckhardts ebenfalls zu Ehren ${ }^{48}$. Grundsätzlich sah er jeden Patienten als therapierbar $\mathrm{an}^{49}$.

Schenkt man den von Burckhardt veröffentlichten Jahresberichten der Anstalt in Préfargier Glauben, so war seine ärztliche Tätigkeit überaus erfolgreich. Als Beispiel soll die Statistik des Jahres 1893 herangezogen werden $^{50}$ : Hiernach konnte die überwiegende Mehrheit der 64 in jenem Jahr aufgenommenen Kranken als geheilt entlassen oder zumindest als «gebessert» eingestuft werden. Lediglich bei 5 dieser Patienten trat nach Aussage von Burckhardt keine Besserung ein; weitere 3 Patienten starben. In jenem Jahr wurden in Préfargier pro Tag und Kopf 4,16 Franken aufgewandt. $\mathrm{Zu}$ Beginn des Jahres zählte die Anstalt 124 Kranke, am Jahresende waren es 128 Patienten.

Nach einer 14jährigen Tätigkeit in Neuchâtel kehrte Burckhardt 1896 nach Basel zurück. Über die Gründe für seinen Weggang gibt es nur Spekulationen. Neben fachlichen Gründen ${ }^{51}$ dürften private Schicksalsschläge eine Rolle gespielt haben, denn Burckhardt verlor im Verlauf seiner Tätigkeit in Neuchâtel sowohl seine Ehefrau als auch einen hoffnungsvollen Sohn ${ }^{52}$. De Meuron stellte in seiner Chronik zur Heilanstalt Préfargier lediglich lapidar fest: «En 1896, le Dr Gottlieb Burckhardt se retira à Bâle, sa ville natale, après avoir assumé la direction de Préfargier pendant 14 ans.» ${ }^{53}$

In seiner Geburtsstadt Basel fand Burckhardt jedoch schon bald eine neue Herausforderung. Er erhielt das Angebot, an der Planung und Einrichtung der Heilanstalt «Sonnenhalde» in Riehen bei Basel mitzuwirken. Als jene Anstalt im Jahr 1900 eröffnet wurde, erklärte Burckhardt sich zudem bereit, die ärztliche Direktion zu übernehmen. Bis zum Sommer 1904 blieb er Leiter der Anstalt. Von 1904 bis zum Ende des Jahres 1906 stellte er seine Dienste noch als konsultierender Arzt zur Verfügung, um sich dann aus dem Berufsleben zurückzuziehen.

47 Bach, a. O. (oben Anm. 19) 531.

48 Bach, a. O. (oben Anm. 19) 532.

49 Bach, a. O. (oben Anm. 19) 531: «Er konnte es nicht sehen, wenn man einen Kranken, weil in der Hauptsache nicht zu helfen war, unbehandelt liess, und fand, man könne immer etwas tun ...»

50 Gottlieb Burckhardt, «Maison de Santé de Préfargier pro 1893», Allgemeine Zeitschrift für Psychiatrie und psychisch-gerichtliche Medizin 51 (1895) 263*.

51 Burckhardts Topektomien fanden im Kollegenkreis nicht die erhoffte Akzeptanz: vgl. hierzu Teil 2 dieses Beitrages.

52 Müller, Father of Topectomy (oben Anm. 2) 463; Bach, a. O. (oben Anm. 19) 529f.; Hagenbach, a. O. (oben Anm. 17) 259.

53 De Meuron, La maison de Santé de Préfargier, Neuchâtel 1949, 69. 
Am 2. Februar 1907 - also nur wenige Wochen nach seinem Abschied von der ärztlichen Tätigkeit - starb Burckhardt plötzlich und unerwartet an den Folgen einer Magenblutung und einer Pneumonie.

In den letzten Lebensjahren hatte Burckhardt keine grösseren wissenschaftlichen Aufsätze mehr veröffentlicht. Der 1891 erschienene Beitrag zur Rindenexzision bei psychotischen Patienten blieb die letzte umfassende Publikation. Sie sollte seine bekannteste, zugleich aber auch seine umstrittenste Arbeit werden (vgl. Abb. 3) $)^{54}$.

\section{Burckhardt und die Psychochirurgie}

Seit der Mitte des 19. Jahrhunderts wurde die Entwicklung der Psychiatrie wie auch die gesamte Medizin - in zunehmendem Masse von den experimentellen Naturwissenschaften beeinflusst. Die Vertreter von Pathologie und Physiologie richteten ihr Augenmerk nunmehr verstärkt auf die Erkrankungen des Gehirns. Paul Broca gelang 1861 die Lokalisation der motorischen Sprachregion ${ }^{55}$. Seine Entdeckung trug massgeblich zur Entwicklung einer anatomisch-lokalisatorischen Psychiatrie bei. Psychische Störungen wurden mehr und mehr als Folge einer Organerkrankung des Gehirns gedeutet.

Einzelne Wissenschaftler gingen dazu über, im Rahmen von Tierversuchen Teile des Gehirns zu entfernen und die dabei auftretenden Verhaltensänderungen zu notieren. So stellte Friedrich Goltz ${ }^{56}$ im Rahmen von Versuchen an Hunden fest, dass diese nach der Entfernung der beiden äusseren Manteloberflächen alle emotionalen Regungen eingebüsst hatten. Aus den Hunden waren «wandernde, fressende und saufende Reflexmaschinen» geworden ${ }^{57}$.

54 Nach 1991 veröffentlichte Burckhardt nur noch kleinere Beiträge zur Epilepsie und zur Pseudoeklampsie: vgl. etwa ders., «Zwei operierte Fälle von Epilepsie», Allgemeine Zeitschrift für Psychiatrie und psychisch-gerichtliche Medizin 48 (1891/92) 658-661.

55 Pierre Paul Broca (1824-1880). Broca fand heraus, dass das motorische Sprachzentrum im unteren Bereich des Gyrus frontalis inferior der dominanten Grosshirnhälfte liegt: ders., «Remarques sur le siège de la faculté du langage articulé, suivie d'une observation d'aphémie (perte de la parole)», Bulletins et memoires de la Societé anatomique de Paris 36 (1861) 330-357, sowie ders., Mémoires sur le cerveau de l'homme et des primates, Paris 1888. Siehe auch Morton's Medical Bibliography (oben Anm. 36) 222.

56 Friedrich Leopold Goltz (1834-1902). Goltz trat vor allem mit neurologischen Untersuchungen an Tieren hervor: vgl. etwa ders., Über die Verrichtungen des Grosshirns, Bonn 1881. Vgl. auch Morton's Medical Bibliography (oben Anm. 36) 214f.

57 Goltz, a. O. (oben Anm. 56) 143; siehe auch Burckhardt, a. O. (oben Anm. 9) 470. 


\title{
Ueber Rindenexcisionen, als Beitrag zur operativen Therapie der Psychosen.
}

\author{
Von \\ Dr. G. Burckhardt, \\ Director in Préfargier.
}

Die folgenden Mittheilungen möchten das Interesse der Herren Collegen für ein Gebiet gewinnen, dass ich vor bald 2 Jahren in der Absicht betreten habe, neue Hilfsmittel fur die Behandlung sonst unheilbarer Psychosen zu finden. Keiner von den operirten Fällen war traumatischen Ursprunges, die Indicationen zur Operation waren deshalb rein psychiatrische. Sie fussen theils auf den anerkannten experimentellen und klinischen Errungenschaften der letzten Jahre, theils auch, ich weiss es wohl, auf eigenen Schlussfolgerungen, deren Berechtigung nicht von vornherein einleuchtet.

Beobachtung 1. Aufgeregte Demenz (chronische Tobsucht), seit $16 \mathrm{~J}$ ahren bestehend, mit heftigen explosivauftretenden Affecten und impulsiven Handlungen mit dem Charakter grosser Gefährlichkeit. 4 sich folgende 0 perationen, Erfolg: Dauernde Beruhigung.

Frau B., A., in Préfargier den 3. Februar 1872 aufgenommen.

Ueber ihr Vorleben, sowie über den Verlauf ihrer Krankheit bis in's Jabr 1882 sind wenig Nachrichten vorhanden - die frühere Krankengeschichte scheint verloren gegangen zu sein. Was ich mittheilen kann, ist Folgendes:

Pat. ist 1837 geboren und hat sich 1857 verheirathet. Der Mann war Trunkenbold, deshalb die Ehe, aus der 3 Kinder stammen, unerfreulich. Pat. lebte in bäuerlichen, ökonomisch ordentlichen Verhältnissen. Sie soll früher gesund gewesen sein - von Vatersseite ist sie hereditär veranlagt. Im Sommer 1871 sollen gastrisch-nervöse Beschwerden aufgetreten sein, Zeitschrift f. Psychiatıie XLV11. 5.

32

Abb. 3. Titelblatt des umstrittenen Artikels «Ueber Rindenexcisionen, als Beitrag zur operativen Therapie der Psychosen» von Gottlieb Burckhardt (1891). 
Burckhardt studierte die Veröffentlichungen von Paul Broca und Friedrich Goltz, aber auch die wissenschaftlichen Beiträge von David Ferrier ${ }^{58}$, Theodor Meynert ${ }^{59}$ und Carl Wernicke ${ }^{60}$. Albert Mairets Theorie, wonach die Temporalwindungen bei Patienten mit akustischen Halluzinationen hypertrophiert seien, hinterliess auf Burckhardt ebenfalls einen tiefen Eindruck ${ }^{61}$. Gleiches gilt für die Arbeiten der Pathologin Leonore Welt, die 1888 im Rahmen einer umfangreichen Studie über «Charakterveränderungen des Menschen infolge von Läsionen des Stirnhirns» feststellte, dass mesio-orbitale Läsionen beim Menschen zu grundlegenden Persönlichkeitsveränderungen führten ${ }^{62}$.

Unter Berufung auf die Ergebnisse der oben genannten Wissenschaftler, aber auch unter Einbeziehung der «eigenen Schlussfolgerungen ${ }^{63}$ entschied sich Burckhardt, bei einigen seiner Patienten hirnchirurgische Eingriffe vorzunehmen. Jener Entschluss muss schon deshalb überraschen, weil die Klinik in Préfargier nur einen kleinen Operationsraum «mit primitiver Einrichtung» besass ${ }^{64}$. Obgleich Burckhardt keinerlei chirurgische Erfahrung aufwies, führte er jene Operationen selbst durch. Die erforderliche Technik erlernte Burckhardt nach eigenen Angaben von seinem Freund, dem Chirurgen und Universitätsprofessor August Socin ${ }^{65}$.

Zwischen 1888 und 1890 entschloss sich Burckhardt zu Operationen an mindestens sechs Patienten. Er ging dabei von der Annahme aus, dass gezielte Rindenexzisionen die Symptomatik der Patienten vermindern oder aufheben könnten. In allen sechs Fällen zielte Burckhardt auf eine Ruhig-

58 David Ferrier (1843-1928). Ferrier arbeitete vor allem über die Lokalisation der zerebralen Funktionen: ders., Die Funktionen des Gehirns, Braunschweig 1879; ders., Die Localisation der Hirnerkrankungen, Braunschweig 1880. Vgl. auch Morton's Medical Bibliography (oben Anm. 36) 224.

59 Theodor Hermann Meynert (1833-1892). Meynert beschrieb u.a. den Bau der Grosshirnrinde und anderer Hirnstrukturen: vgl. ders., Theodor Meynert, Psychiatry: A clinical treatise on diseases of the fore-brain based upon a study of its structure, functions and nutrition, with a new introduction by Stanley W. Jackson, Reprint New York 1968. Siehe auch Morton's Medical Bibliography (oben Anm. 36) 222, sowie Kolle, a. O. (oben Anm. 36), Bd. 2, 98-105.

60 Carl Wernicke (1848-1905). Wernicke führte massgebliche Untersuchungen zur sensorischen Aphasie durch: ders., Der aphasische Symptomenkomplex, Breslau 1874. Vgl. auch Morton's Medical Bibliography (1991) 716; Kolle, a. O. (oben Anm. 36), Bd. 2, 106-128.

$61 \mathrm{Vgl}$. Albert Mairet, De la démence mélancolique: Contribution à l'étude de la périencéphalite localisée, et à l'étude des localisations cérébrales d'ordre psychique, Paris 1883.

62 Leonore Welt, «Über Charakterveränderungen des Menschen infolge von Läsionen des Stirnhirns», Deutsches Archiv für Klinische Medicin 42 (1888) 339-390.

63 Burckhardt, a. O. (oben Anm. 9) 463.

64 Müller, Tollhaus (oben Anm. 2) 144.

65 August Socin (1837-1899). Socin hatte u.a. in Basel und Würzburg studiert. Nach seiner Promotion in Würzburg (1857) habilitierte er sich 1861 in Basel, wo er 1864 ordentlicher Professor der Chirurgie wurde. Socin machte sich vor allem um die Verbesserung des chirurgischen Unterrichts in Basel verdient: vgl. auch Biographisches Lexikon der hervorragenden Ärzte aller Zeiten und Völker, hg. von August Hirsch, Bd. 5, Berlin ${ }^{21934, ~ 327 ; ~ T e i c h m a n n, ~}$ a. O. (oben Anm. 16) 50. 
stellung der betreffenden Kranken ab; eine Heilung hielt er selbst für ausgeschlossen.

Nachfolgend sollen die sechs überlieferten Kasuistiken en détail vorgestellt werden. Um Burckhardts Ansichten und Einschätzungen möglichst unverfälscht wiederzugeben, werden hierbei die entscheidenden Stellungnahmen wörtlich zitiert.

\section{Fallbeschreibung}

Bei der Übernahme der Anstaltsleitung im Jahr 1882 sah sich Burckhardt mit einer Patientin konfrontiert, die trotz Zwangsmassnahmen Symptome einer «chronischen Tobsucht» ${ }^{66}$ zeigte und zu extremer Gewalttätigkeit neigte ${ }^{67}$. Es handelte sich um eine Mutter von drei Kindern, die mit einem «Trunkenbold» verheiratet war und vor ihrer Erkrankung in «bäuerlichen, ökonomisch ordentlichen Verhältnissen» gelebt hatte ${ }^{68}$. Sie war bereits $1872 \mathrm{im}$ Alter von 35 Jahren in die Anstalt eingewiesen worden und galt Burckhardt zufolge als «gefährlichste und in jeder Beziehung ärgste Patientin der Abteilung ${ }^{69}$. Freeman und Watts beschrieben die Patientin 1942 retrospektiv als «the worst type of schizophrenic» ${ }^{70}$.

Burckhardt warf in seiner Veröffentlichung zunächst die Frage auf, ob «man nicht dieses emotivimpulsive Element aus ihrem Gehirnmechanismus wegnehmen und dadurch die Pat. aus einer aufgeregten $\mathrm{zu}$ einer ruhigen Dementen machen» könnte. Zwar wäre die betreffende Person danach immer noch «dement, unreinlich u.s.w, hätte aber überall können verpflegt werden ${ }^{71}$. Er nahm an, dass die Agitiertheit und Impulsivität der Patientin durch starke Stimuli in der sensorischen Region hervorgerufen würden, die dann in die motorische Region überträten. Daher fasste er den Entschluss, die Assoziationsfasern zwischen dem sensorischen und dem motorischen Areal zu durchtrennen. Am 29. Dezember 1888 wurde die nunmehr 51jährige Frau als erste Patientin überhaupt einem psychochirurgischen Eingriff unterzogen. Burckhardt entfernte nach der Rasur des Schädels und dem Anbringen von Bohrlöchern in einer vierstündigen Operation im lateralen Teil des oberen Parietallappens der linken Hemisphäre rund 5 Gramm der Hirnrinde ${ }^{72}$.

66 Burckhardt, a. O. (oben Anm. 9) 463.

67 Burckhardt, a. O. (oben Anm. 9) 467.

68 Burckhardt, a. O. (oben Anm. 9) 463.

69 Burckhardt, a. O. (oben Anm. 9) 464.

70 Freemann/Watts, a. O. (oben Anm. 15) 7.

71 Burckhardt, a. O. (oben Anm. 9) 465-468, insb. 468 (Zitat).

72 Burckhardt, a. O. (oben Anm. 9) 479. 
Nach dem Eingriff traten bei der Patientin zunächst febrile Temperaturen und vorübergehende Lähmungserscheinungen auf ${ }^{73}$. Während die Tobsuchtsanfälle postoperativ abnahmen, gewannen die Halluzinationen an klinischer Relevanz, so dass Burckhard sich entschloss, in einer Folgeoperation am 8. März 1889 einzelne Exzisionen im Bereich des Temporallappens vorzunehmen. In einer zweieinhalbstündigen Operation wurden 2,5 Gramm kortikale Hirnsubstanz entfernt ${ }^{74}$.

Obwohl die Patientin nach der zweiten Operation insgesamt weniger depressiv schien, litt sie doch immer noch an plötzlichen Stimmungsschwankungen, so dass Burckhardt sich zu einem dritten Eingriff entschloss $^{75}$. Am 29. Mai 1889 nahm er Rindenexzisionen im Bereich des linken Parietallappens vor; in einer viereinhalbstündigen Operation wurden weitere 5,5 Gramm Hirnrinde entfernt. Nunmehr stellte Burckhardt eine «Besserung» ihres Zustandes fest: «Immerhin fällt uns angenehm auf, dass Pat. viel weniger Unanständigkeiten sagt.» Allerdings litt die Patientin an Logorrhoe, woraus Burckhardt schloss, «dass die Gegend der motorischen Sprachcentren das nächste Angriffsobject bilden müsse» ${ }^{76}$.

Nachdem Burckhardt am 12. Februar 1890 in einer vierten, zweieinhalbstündigen Operation aus der linken dritten Frontalwindung zusätzliches Hirngewebe exzidiert hatte, gab er sich mit dem Ergebnis seiner operativen Tätigkeit zufrieden. Als Erfolgsparameter diente ihm die (Lärm)belästigung durch die Patientin:

«Seit vielen Wochen ist auch nicht die leiseste Störung vorgekommen. Man hört absolut nichts mehr von Frau Borel ... Sie wird weder von anderen Kranken noch von Wärterinnen mehr gefürchtet; sie wird im Gegentheil verhätschelt; die Wärterinnen behandeln sie wie das Kind der Abteilung.» ${ }^{77}$

\section{Fallbeschreibung}

Bei dem zweiten von Burckhardt operierten Patienten handelte es sich um einen 31jährigen, als gemeingefährlich beschriebenen Lithographen. Der betreffende Kranke sollte «sich als Schüler begabt gezeigt, aber schon früher onaniert und sehr frühe in Venere und Baccho excediert haben $»^{78} .1884$ war der Patient in die Anstalt eingewiesen worden. Burckhardt stellte die Dia-

73 Burckhardt, a. O. (oben Anm. 9) 480-482.

74 Burckhardt, a. O. (oben Anm. 9) 482-483.

75 Burckhardt, a. O. (oben Anm. 9) 484 («Die Emotivität ist ungefähr gleich»).

76 Burckhardt, a. O. (oben Anm. 9) 490.

77 Burckhardt, a. O. (oben Anm. 9) 492.

78 Burckhardt, a. O. (oben Anm. 9) 494. 
gnose einer primär entstandenen, chronisch gewordenen Demenz, «welche den Pat. durch ihre immer noch vorhandenen Restzustände zu einem lästigen ja gefährlichen Menschen macht» ${ }^{79}$.

Der Patient wurde am 17. April 1889 operiert. Ziel des Eingriffes war es, «die aufgeregte Demenz in eine ruhigere event. ruhige überzuführen» ${ }^{80}$. In der dreistündigen Operation nahm Burckhardt Exzisionen im Bereich des Stirnhirns vor ${ }^{81}$. Der Eingriff hatte starke epileptoide Anfälle zur Folge, so dass Burckhardt von einer bereits geplanten zweiten Intervention zunächst absah. Er räumte ein, dass derartige Anfälle zuvor nie bemerkt worden seien. Allerdings vertrat er die Ansicht, dass eine Disposition bestanden haben könnte, die durch den operativen Eingriff «zum Ausbruch gebracht» wurde ${ }^{82}$. Sein vorläufiges Resümee fiel dennoch positiv aus:

«Ich muss sagen, dass ich relativ nicht unzufrieden wäre, sollte die weitere Beobachtung den corticalen Charakter und also auch Ursprung der Anfälle bestätigen. - Denn es dürfte dann auf eine locale Ursache geschlossen werden, etwa auf Narbenzug oder Derartiges, und das Heilmittel wäre in der Entfernung der Ursache gegeben ... Ganz ohne Gewinn scheint mir trotzdem die Operation nicht gewesen zu sein. Sie hat zunächst, wie ich glaube, den Zusammenhang von Gehörstäuschungen und explosiven Handlungen deutlicher herausgestellt, als es vorher vorausgesetzt werden konnte, und hat somit einen Fingerzeig für das weitere Verfahren gegeben. $»^{83}$

\section{Fallbeschreibung}

Nur eine Woche später operierte Burckhardt einen 35jährigen halluzinierenden Gärtner, der seit 1885 in Préfargier stationär behandelt worden war. Als Diagnose nannte Burckhardt eine «chronische, wahrscheinlich originäre Verrücktheit» ${ }^{84}$; als Indikation für den Eingriff führte er die Tatsache an, dass der Patient wegen «Gefährlichkeit» öfters isoliert werden musste ${ }^{85}$. Wiederum gestand der Operateur ein: «Meine Absicht konnte auch in diesem Fall keine andere sein, als aus dem gefährlichen Kranken einen ungefährlichen zu machen.» ${ }^{86}$

Burckhardt wollte vor allem gegen die akustischen Halluzinationen angehen und dachte über einen Eingriff im Brocaschen Sprachzentrum nach. Dabei liess er sich von der Annahme leiten, «dass erst durch diese Mit-

79 Burckhardt, a. O. (oben Anm. 9) 495.

80 Burckhardt, a. O. (oben Anm. 9) 496.

81 Burckhardt, a. O. (oben Anm. 9) 496f.

82 Burckhardt, a. O. (oben Anm. 9) 501.

83 Ebenda.

84 Burckhardt, a. O. (oben Anm. 9) 502.

85 Burckhardt, a. O. (oben Anm. 9) 504.

86 Burckhardt, a. O. (oben Anm. 9) 505. 
wirkung des darin ruhenden motorischen Elementes die Hallucinationen die Deutlichkeit und Ueberzeugungsstärke des gesprochenen Wortes erhalten ${ }^{87}$. Andererseits fürchtete Burckhardt bei einem Eingriff am BrocaZentrum das Auftreten einer motorischen Aphasie. Alternativ erwog er eine Operation am im Schläfenlappen gelegenen Wernicke-Zentrum ${ }^{88}$; hierbei war jedoch mit einer sensorischen Aphasie zu rechnen. Da Burckhardt einen motorisch bedingten Verlust des Sprechvermögens für die «schwerere Verstümmelung» hielt, entschied er sich letztlich für eine chirurgische Intervention im Bereich des Schläfenlappens.

In einem zweieinhalbstündigen Eingriff entfernte er 2,8 Gramm Hirnrinde. Die Operation hatte keine Aphasie zur Folge.Während der Patient eine deutlich geringere Gewaltbereitschaft zeigte, beklagte Burckhardt jedoch einen Verlust an Sittsamkeit:

«Dagegen hat sich ein erotischer Zug in seinem Wesen bemerklich gemacht ... Doch war er uns schon längere Zeit der Masturbation verdächtig. Jetzt, wo er sich wieder in normalen Geleisen bewegt, tritt jener Zug mehr hervor.» ${ }^{89}$

Insgesamt kam Burckhardt zu dem Schluss:

«Ich halte dafür, dass Pat. weniger von Hallucinationen zu leiden hat als früher ... So erfreulich dieser Erfolg ist, so betrachte ich ihn noch nicht als abgeschlossen.- Ich erhoffe noch Mehreres - und werde eventuell zu einer zweiten Operation schreiten.» ${ }^{90}$

\section{Fallbeschreibung}

Am 7. Juni 1889 nahm Burckhardt einen hirnchirurgischen Eingriff bei einer 37jährigen Witwe vor, bei der er eine «acute primäre Verrücktheit mit Vorwiegen verbaler Gehörshallucinationen» diagnostizierte, die nach zwei Jahren «in aufgeregte Demenz mit Bestehenbleiben der Hallucinationen» übergegangen sei ${ }^{91}$. Obwohl Burckhardt die Patientin als dement beschrieb, hob er hervor, dass sie seinem Vorschlag, «den Hallucinationen durch eine Operation zu Leibe zu gehen», gerne zugestimmt habe ${ }^{92}$. Wiederum nahm er sich das Wernicke-Zentrum als Operationsfeld vor:

87 Burckhardt, a. O. (oben Anm. 9) $507 f$.

88 Hierbei handelt es sich um die von Wernicke beschriebene sensorische Sprachregion im hinteren Bereich des Gyrus temporalis superior der dominanten Hemisphäre.

89 Burckhardt, a. O. (oben Anm. 9) 511.

90 Ebenda.

91 Burckhardt, a. O. (oben Anm. 9) 511f.

92 Burckhardt, a. O. (oben Anm. 9) 515. 
«Es war beabsichtigt, wieder den hinteren Theil der 1. und die mediale Hälfte des 2. T.-W. ${ }^{93}$, also einen Theil des sensoriellen Wortgedächtnisses zu entfernen.» ${ }^{94}$

Während des knapp dreistündigen Eingriffs entfernte er insgesamt 3 Gramm Hirnrinde. Im Unterschied zum vorangegangenen Patienten zeigte sich bei der Witwe «ein gewisser Grad von Aphasie» ${ }^{95}$. So gab Burckhardt an:

«Pat. verwechselte Wörter, besonders Namen von Gegenständen, oder wusste sie überhaupt nicht zu benennen, z.B. Schere, Uhr. Sie sprach zögernd, in kurzen Sätzen, oft sich unterbrechend. Manchmal kamen die Wörter undeutlich heraus, mehr oder weniger unvollständig, oder die Wörter waren gut gebildet, aber unrichtig angewandt.» ${ }^{96}$

Zudem fühlte sich die Patientin «gelegentlich unglücklich und geplagt, aber sie beschuldigt kaum mehr diese oder jene Person, dies oder jenes über sie gesagt zu haben». Burckhardt deutete dies «im Sinne einer Abnahme der Hallucinationen $»^{97}$.

Im Herbst 1889 schien sich ihr Befinden zu bessern. Wohl auf Wunsch ihrer Schwester wurde die Patientin am 21. November 1889 entlassen. Schon am 28. Dezember verschwand sie spurlos; zwei Tage später wurde ihre Leiche gefunden. Nach Aussagen von Burckhardt blieb ungeklärt, ob der plötzliche Tod durch einen Suizid oder einen Unfall verursacht war. Eine Fremdeinwirkung konnte indessen ausgeschlossen werden ${ }^{98}$.

\section{Fallbeschreibung}

Bei dem fünften von Burckhardt operierten Patienten handelte es sich um einen 26jährigen Kunstmaler, bei dem eine «chronisch gewordene primäre Verrücktheit» diagnostiziert wurde ${ }^{99} .1886 / 87$ fiel er «durch unruhiges, unstätes Wesen auf, durch perverse Behauptungen, z. B. er sei der Sohn eines Fürsten» ${ }^{100}$. Als Depressionen und akustische Halluzinationen auftraten, wurde er im Juli 1887 nach Préfargier überwiesen ${ }^{101}$. Zu den akustischen Halluzinationen gesellten sich optische hinzu. Zu Beginn des Jahres 1889 verschlechterte sich sein Zustand zusehends. Hierzu stellte Burckhardt fest:

93 T. W. $=$ Temporal-Windung.

94 Burckhardt, a. O. (oben Anm. 9) 515.

95 Burckhardt, a. O. (oben Anm. 9) 517.

96 Ebenda.

97 Ebenda.

98 Burckhardt, a. O. (oben Anm. 9) 519.

99 Burckhardt, a. O. (oben Anm. 9) 520.

100 Burckhardt, a. O. (oben Anm. 9) 521.

101 Ebenda. 
«Er legte sich keinerlei Rückhalt mehr auf, sondern berichtete mit lauter Stimme und ohne Unterlass den Inhalt seiner sehr starken erotischen Hallucinationen. Es war schon aus diesem Grunde nicht mehr möglich, ihn auf den Abtheilungen der ruhigen Patienten zu behalten ... Er gehorchte nicht mehr. Dies hatte zur Folge, dass er Nachts öfters musste isolirt werden, weil er für die Wachabteilung, in welche sein Zimmer ging, und wo er sich schon während seines zweiten Aufenthaltes befunden hatte, zu störend wurde. Auch Tags über war er lästig genug.»102

Nach einer am 5. Juni 1889 durchgeführten mehr als dreistündigen Operation im Bereich des Wernicke-Zentrums ${ }^{103}$ verhielt sich der Patient zunächst ruhig. Laut Burckhardt «war eine höchst wohlthätige Stille in seinem Zimmer und der anstossenden Wachstation eingekehrt» ${ }^{104}$. Obwohl der ehemalige Maler weiterhin Stimmen vernahm, waren «die Obscönitäten bedeutend weniger geworden» ${ }^{105}$.

Nach einiger Zeit fing er jedoch «wieder etwas lebhafter zu sprechen» an, so dass Burckhardt sich zu einer neuerlichen Operation entschloss ${ }^{106}$. Der Folgeeingriff - diesmal am Brocaschen Sprachzentrum, also im Bereich des Frontallappens - wurde am 19. Februar 1890 durchgeführt und dauerte noch einmal zweieinhalb Stunden. Hatte Burckhardt bei der ersten Operation 4,6 Gramm kortikales Gewebe entfernt, so resezierte er nun weitere 2,5 Gramm.

Der Eingriff zeigte den von Burckhardt gewünschten Erfolg:

«Pat. spricht seit der 2. Operation nicht nur viel weniger, sondern auch leiser als früher. Er ist auch sonst ruhiger ... Manchmal nimmt er auch gar keine Notiz von mir; er macht sich nicht mehr bemerklich oder lästig, wie früher ... Dieser Eindruck, dass Herr M. viel ruhiger ist als früher, manierlicher, anständiger, lenksamer, ist ein allgemeiner. Seine Mutter hat mir ihn bei ihren letzten Besuchen wiederholt und ausdrücklich bestätigt.»107

In demselben Masse, in dem der Wortschatz des 26jährigen abnahm, gingen auch die Halluzinationen zurück. Bemerkenswerterweise traten bei dem Patienten keine Aphasien auf. Ein Verfehlen der Brocaschen Region schloss Burckhardt jedoch aus. Vielmehr führte er eine latente «theilweise Linkshändigkeit» und eine stärkere Ausdehnung der Sprachregion als mögliche Erklärungen $a^{108}$. Dementsprechend spielte Burckhardt mit dem Gedanken an eine zweite Operation im Bereich des rechten Frontalhirns: «Ich werde mich dann so einrichten, ein grösseres Stück der Rinde und eventuell der weissen Substanz mitzunehmen.» ${ }^{109}$ Francois Moutier berief sich

102 Burckhardt, a. O. (oben Anm. 9) 525.

103 Burckhardt, a. O. (oben Anm. 9) 528.

104 Ebenda.

105 Burckhardt, a. O. (oben Anm. 9) 529.

106 Ebenda.

107 Burckhardt, a. O. (oben Anm. 9) 532.

108 Burckhardt, a. O. (oben Anm. 9) 532f.

109 Burckhardt, a. O. (oben Anm. 9) 533. 
übrigens später auf jene Fallbeschreibung Burckhardts, als er behauptete, dass die Brocasche Region keine spezielle Bedeutung für die Sprache habe ${ }^{110}$.

\section{Fallbeschreibung}

Die sechste und letzte von Burckhardt beschriebene Kasuistik betraf einen 33jährigen ehemaligen Uhrmacher, der erst im November 1889 in die Anstalt aufgenommen worden war. Auch bei ihm stellte Burckhardt eine «Primäre Verrücktheit, mit starker Entwickelung verbaler Gehörshallucinationen» fest. Zudem beschrieb er «gefährliche Abwehrhandlungen» ${ }^{111}$.

Die präoperativen Untersuchungen gestalteten sich indessen schwieriger als sonst. In der Krankenakte hielt Burckhardt fest:

«Die Vorbereitungen zur Operation beunruhigen ihn. Er fürchtet, man wolle ihm den Kopf in einen eisernen Helm pressen. Pat. hielt sich sehr unruhig, so dass die Messung schwierig war, und mir trotz mehrmaliger Wiederholung nicht zuverlässig schien. Deswegen wurde sie gerade vor der Operation nochmals wiederholt.»112

Burckhardt strebte eine «ausgiebigere Zerstörung des acustischen Wortfeldes» an. Hierzu zielte er auf die erste und zweite Temporalwindung ab. Die Operation erfolgte am 9. April 1890 und verlief nicht ohne Zwischenfälle ${ }^{113}$ : Nach der Eröffnung des Schädels mit dem Meissel musste Burckhardt feststellen, «dass sich die Dura in Folge der Hammerschläge mit feinen punktförmigen Hämorrhagien bedeckte, und dass die Gehirnoberfläche (soweit sie sichtbar war) ein schiefriges Ansehn annahm» ${ }^{114}$. Daher sah er von einem weiteren Gebrauch des Meissels ab. Nach dreieinhalb Stunden war der Eingriff beendet; Burckhardt hatte 3,4 Gramm Rindensubstanz entfernt.

Postoperativ stellten sich bei dem Patienten eine sensorische Aphasie, aber auch eine ataktische Aphasie und Agraphie ein. Burckhardt notierte:

«Die Worttaubheit ist vollständig. Die Verminderung des Sprachschatzes ziemlich gross ... Schriftlich ist nicht mit ihm zu verkehren, vielleicht versteht er die Schriftsprache auch nicht, oder er concentrirt seine Aufmerksamkeit nicht; er ist überhaupt wie in einem fremden Land.» ${ }^{115}$

Am sechsten Tag post operationem verstarb der Patient ${ }^{116}$. Die Autopsie ergab das Bild einer Paralyse der Zerebralgefässe. Burckhardt erklärte jenen

110 Francois Moutier, L'aphasie de Broca, Paris 1908.

111 Burckhardt, a. O. (oben Anm. 9) 533.

112 Burckhardt, a. O. (oben Anm. 9) 537.

113 Ebenda.

114 Burckhardt, a. O. (oben Anm. 9) 538.

115 Burckhardt, a. O. (oben Anm. 9) 540.

116 Burckhardt, a. O. (oben Anm. 9) 542. 
Befund mit der «Erschütterung ..., welche das Meisseln verursachte; allerdings bei einer besonderen idiosyncratischen Beschaffenheit des Gehirns, welche nicht vorauszusehen war» ${ }^{117}$.

Es handelte sich vermutlich um den letzten von Burckhardt operierten Patienten, sicher jedoch um den letzten öffentlich gemachten Fall.

\section{Burckhardts Beitrag zur Psychochirurgie kritisch betrachtet}

Burckhardt war sich durchaus bewusst, dass die Bekanntmachung seiner Topektomien eine Kontroverse auslösen würde. Dies ergibt sich bereits aus der Tatsache, dass er in seiner oben zitierten Publikation mögliche Einwände seiner Gegner vorwegnahm und zu entkräften suchte. Zudem schloss er den Fallberichten eine Diskussion seiner Vorgehensweise und seiner Ergebnisse an. Das erste Augenmerk soll daher der Argumentationsweise Burckhardts gelten:

Wie er in seiner Veröffentlichung feststellte, fussten die Indikationen zur Operation «theils auf den anerkannten experimentellen und klinischen Errungenschaften der letzten Jahre, theils auch, ich weiss es wohl, auf eigenen Schlussfolgerungen, deren Berechtigung nicht von vornherein einleuchtet» ${ }^{118}$. Burckhardt räumte ein, dass die Begründung des operativen Eingriffs nur «eine wissenschaftliche sein» könne, «und da werden die Ansichten zweifelsohne ziemlich weit auseinander gehen» ${ }^{119}$. Im Hinblick auf die Ursache der Psychosen unterschied er zwei Erklärungsmodelle. Er selbst stand auf dem Standpunkt, «dass sich auch unser psychisches Leben aus einzelnen Elementen aufbaut, die sich im Gehirn örtlich getrennt ansetzen ...». Manche Ärzte hielten dagegen «an der prästabilirten Einheit unseres psychischen Seins als einer unauflöslichen» fest. Für einen solchen Kollegen würde «es selbstverständlich keinen Sinn haben, relativ kleine Stücke aus der Rinde auszuschneiden, in der Hoffnung, dadurch eine Psychose günstig zu beeinflussen ${ }^{120}$. Nachfolgend führte Burckhardt verschiedene Studien an, in denen er seine Auffassung bestätigt sah, um schliesslich festzustellen:

«Folgerichtig würden wir die Psychosen nicht als diffuse Erkrankungen der Rinde, sondern als solche ansehen, welche eine mehr oder minder grosse Zahl von Gliedern in der Kette des psych. Geschehens, resp. von einzelnen Rindenherden ergreifen, wobei der Ausgangspunkt und Ausbreitung verschiedene sind, und folglich die Erscheinungen auch. Nur so erklärt es sich, dass verschiedenartige Delirien gleichzeitig bestehn können, eine Thatsache, die mehr und mehr Zustimmung findet ...» ${ }^{121}$

117 Burckhardt, a. O. (oben Anm. 9) 544.

118 Burckhardt, a. O. (oben Anm. 9) 463.

119 Burckhardt, a. O. (oben Anm. 9) 544.

120 Ebenda.

121 Burckhardt, a. O. (oben Anm. 9) 546. 
Auch für die Risiken seiner Therapie glaubte Burckhardt eine hinreichende Rechtfertigung gefunden zu haben:

«Jeder neue chirurgische Eingriff hat sich seine speciellen Indicationen und Methoden erst suchen müssen und jeder Weg, der zu neuen Errungenschaften geführt hat, ist mit Todtenkreuzen besteckt. Ich glaube aber nicht, dass wir uns deswegen sollen abhalten lassen, dem vorgesteckten Ziele, der Heilung unsrer Kranken durch die operative Behandlung, näher zu kommen.» ${ }^{122}$

\section{Schliesslich zog Burckhardt Bilanz:}

«Aber wird man schliesslich fragen, wiegen die erzielten Erfolge die Gefahren auf, denen die Operirten ausgesetzt werden? So bescheiden meine bisherigen Resultate sind, so glaube ich doch, dass sie gegenüber der Hoffnungslosigkeit der operirten Kranken einen Fortschritt darstellen. Die Fälle I, III und V halte ich für Resultate, die anderswie nicht hätten erreicht werden können. Fall IV glaube ich ebendahin rechnen zu dürfen. Fall II zeigt eine partielle Besserung, bleibt aber weiterer Behandlung gewärtig. Ich hatte gehofft, vom letzten Falle ein besonders erfreuliches definitives Resultat mittheilen zu können. Es hat nicht sein sollen. Aber ich selbst werde mich nicht entmutigen lassen, und hoffe, die Collegen werden es auch nicht thun, sondern werden, meine Erfahrungen benutzend, selbst den Weg der Rindenexstirpationen betreten und immer bessere und vollkommenere Resultate erzielen.» ${ }^{123}$

Um das Vorgehen Burckhardts richtig einordnen zu können, ist zu berücksichtigen, dass die Situation psychotischer Anstaltsinsassen zu seiner Zeit geradezu trostlos war. Angesichts der geringen therapeutischen Möglichkeiten konnte jeder noch so verwegene neue Behandlungsversuch einen möglichen Ausweg darstellen.

Andererseits lässt Burckhardt - zumindest aus heutiger Sicht - die notwendige Selbstkritik vermissen. So stellt sich die Frage, an welchen Erfolgskriterien dieser die Einstufung als «erfreuliches Resultat» einer Behandlung orientiert. Objektivierbare und verbindliche Parameter, anhand derer sich ein Erfolg feststellen liesse, nennt er nicht. Eine Heilung der Patienten lag nach eigenem Bekunden ohnehin ausserhalb der therapeutischen Möglichkeiten. Wie Burckhardt selbst konzediert, wurden bei den Kranken lediglich eine Verminderung der Gewalttätigkeit und des durch den Patienten verursachten Betreuungsaufwandes sowie die Wiederherstellung der «Sittsamkeit» angestrebt. Ein weiteres Kriterium Burckhardts scheint das Ausbleiben operationsbedingter Folgeschäden zu sein ${ }^{124}$.

In der Tat beschreibt er einige seiner Patienten postoperativ als ruhiger, manierlicher und lenksamer. Nicht die Heilung von einer psychischen Krank-

122 Burckhardt, a. O. (oben Anm. 9) 547.

123 Burckhardt, a. O. (oben Anm. 9) 547f.

124 Burckhardt, a. O. (oben Anm. 9), etwa S. 494 («Ihre Intelligenz hat sie nicht zurückerlangt. Sie hat aber entschieden nichts von dem, was sie noch hatte, eingebüsst.») u. S. 511 («Dement ist er geblieben. - Doch hat sich die Demenz entschieden nicht vergrössert, in manchen Punkten dagegen verringert.») 
heit, sondern die Verbesserung der gesellschaftlichen Konformität und die Schaffung eines pflegeleichten Kranken waren die eigentlichen Intentionen der Eingriffe. Paradigmatisch für diese Sicht ist Burckhardts Resümee bezüglich seines ersten «Falls»:

«Frau Borel ist also aus einer gefährlichen und aufgeregten Dementen eine ruhige Demente geworden. - Der beständig fliessende Redefluss ist unterbrochen.» ${ }^{125}$

Doch selbst wenn man Burckhardts Kriterien - die Ruhigstellung des Kranken und das Ausbleiben operationsbedingter Sekundärschäden akzeptiert, scheint seine Bilanz geschönt: In vier von sechs Fällen zieht Burckhardt ein positives Fazit, in einem Fall macht er einen Teilerfolg geltend und im letzten Fall führt er höhere Gewalt $a^{126}$. Der Leser seiner Kasuistiken gewinnt den Eindruck, dass Burckhardt hierbei einige wesentliche Details übersehen hat. So traten beim zweiten Patienten postoperativ erstmals epileptische Anfälle auf, die vorher «nie bemerkt worden» waren ${ }^{127}$. Beim dritten Patienten machten sich nach der Operation «ein erotischer Zug in seinem Wesen bemerkbar», den Burckhardt selbst - ob zu Recht oder zu Unrecht - negativ bewertete ${ }^{128}$. Die vierte Patientin kam nur wenige Wochen nach ihrer (vorzeitigen?) Entlassung unter ungeklärten Umständen ums Leben, und der im sechsten Fallbericht beschriebene Uhrmacher starb zweifelsfrei an den Folgen der Operation. Allenfalls der erste und der fünfte Fallbericht können unter Zugrundelegung von Burckhardts Kriterien als Erfolg eingestuft werden - wenngleich er bei den betroffenen Patienten vier bzw. zwei Operationen benötigte, um schliesslich zu dem gewünschten Ergebnis zu kommen.

Neben der mangelnden Selbstkritik fällt die häufig recht unglückliche Wortwahl Burckhardts auf: So bezeichnet er etwa die Patienten durchgehend als «Fälle» oder «Beobachtungen», Zielpunkte im Gehirn tituliert er militärisch als «Angriffsobjecte», und das präoperative Verhalten der betroffenen Kranken nennt er verschiedentlich «lästig» ${ }^{129}$.

Nach der Auseinandersetzung mit der Argumentationsweise Burckhardts ist zu fragen, welche Reaktionen seine Topektomien unter den Kollegen hervorriefen:

125 Burckhardt, a. O. (oben Anm. 9) 493. Berücksichtigt man jedoch, dass die beschriebenen Operationen im wesentlichen auf eine Ruhigstellung der Patienten abzielten, so hinterlässt seine oben zitierte Feststellung, jeder Weg «zu neuen Errungenschaften» sei mit «Todtenkreuzen besteckt», einen bitteren Beigeschmack: vgl. Burckhardt, a. O. (oben Anm. 9) 547.

126 Burckhardt, a. O. (oben Anm. 9) 548 («Es hat nicht sein sollen»).

127 Burckhardt, a. O. (oben Anm. 9) 500.

128 Burckhardt, a. O. (oben Anm. 9) 511.

129 Vgl. Burckhardt, a. O. (oben Anm. 9), etwa 490, 495 u. 525. 
Mit welchen Worten Gottlieb Burckhardt seine Therapieversuche auf dem Berliner Kongress für Psychiatrie (1890) vorstellte und kommentierte, ist nicht überliefert. Jedenfalls verursachten seine Ausführungen ein Frösteln unter den Kollegen; die erhoffte Zustimmung blieb aus ${ }^{130}$. Neben dem beschriebenen privaten Unglück ${ }^{131}$ dürfte jene fehlende Akzeptanz seines Vorgehens ursächlich sein für die Tatsache, dass Burckhardt seine Operationsmethoden entgegen seiner Ankündigung ${ }^{132}$ nicht weiterführte oder doch zumindest von einer Veröffentlichung weiterer Eingriffe absah ${ }^{133}$.

Im angloamerikanischen Sprachraum wurden Burckhardts psychochirurgische Ambitionen zunächst schlichtweg nicht zur Kenntnis genommen. Aber auch im deutschen Sprachgebiet wurde sein für die damalige Zeit ungeheuerlicher Vorstoss weitgehend mit Nichtachtung bestraft. Selbst in den drei verfügbaren Nachrufen auf Burckhardt werden dessen Aktivitäten im Bereich der Psychochirurgie mit auffälliger Zurückhaltung behandelt:

C. Bach, Burckhardts Kollege und Nachfolger in der Heilanstalt Sonnenhalde, nimmt in dem ansonsten sehr freundlich formulierten Nekrolog in der Allgemeinen Zeitschrift für Psychiatrie nur in zwei knappen Sätzen auf jene Eingriffe Bezug. Die mehrdeutige Bemerkung Bachs, Burckhardt habe «in kühner Weise das Gebiet der Gehirnchirurgie» beschritten, lässt die Distanz zu jenen Operationen erahnen. Eine Erwähnung oder gar Würdigung der Tatsache, dass Burckhardt als erster Arzt überhaupt einen psychochirurgischen Eingriff vorgenommen hatte, unterbleibt $t^{134}$.

Mindestens ebenso aufschlussreich sind die beiden Nekrologe im Korrespondenzblatt für Schweizer Ärzte und in der Psychiatrisch-neurologischen Wochenschrift: Während die verantwortlichen Autoren Hagenbach und Bresler in lobenden Worten auf die wissenschaftliche und die klinischpraktische Tätigkeit Gottlieb Burckhardts Bezug nehmen, bleibt sein Engagement in der Psychochirurgie hier gänzlich unerwähnt ${ }^{135}$.

Fest steht, dass Burckhardts Operationsmethoden zunächst nicht weitergeführt wurden. Zu den wenigen Rezipienten seiner psychochirurgischen Studie gehörte François Moutier. Letzterer erwähnte Burckhardts Versuche in seiner Doktorabeit von 1908, ohne jedoch zu den ethischen Implikationen des Vorgehens Stellung zu beziehen ${ }^{136}$.

130 Vgl. auch de Meuron, a. O. (oben Anm. 53); Müller, a. O. (oben Anm. 2) 462f.

131 Wie oben erwähnt, verlor Burckhardt während seiner 14jährigen Tätigkeit in Préfargier seine Frau und seinen Sohn: Bach, a. O. (oben Anm. 19) 529f.

132 Burckhardt, a. O. (oben Anm. 9) 548 («Aber ich selbst werde mich nicht entmutigen lassen ...»).

133 Müller, a. O. (oben Anm. 2) 462f.

134 Bach, a. O. (oben Anm. 19) 531.

135 Hagenbach (oben Anm. 17) 257-260; Bresler (oben Anm. 19) 464.

136 Siehe Moutier (oben Anm. 110). 
Kritisch äusserten sich dagegen der russische Neurologe Wladimir M.von Bechterew ${ }^{137}$ und der estländische Neurochirurg Ludwig M. Puusepp ${ }^{138}$ in einer gemeinsamen Publikation aus dem Jahr 1908:

«One can't but admit the futility of the operations made and mention the marked damage caused by severe cerebral intervention. One can only be surprised the medical doctor's performing such operations. ${ }^{139}$

Bemerkenswerterweise war es jedoch gerade Ludwig M. Puusepp, der zwischen 1906 und 1910 in Dorpat vergleichbare chirurgische Eingriffe bei manischen, depressiven und epileptischen Patienten vornahm. Doch erst in den Dreissigerjahren, als die Psychochirurgie eine gewisse Popularität und Anerkennung erzielt hatte, bekannte sich Puusepp zu seinen damaligen Operationen und erstattete über seine Erfahrungen Bericht. Nunmehr setzte er in Italien seine Hirneingriffe fort ${ }^{140}$.

Die erste Frontalhirn-Leukotomie nahmen 1935 der portugiesische Neurologe und spätere Nobelpreisträger für Medizin Egas Moniz und der Neurochirurg Almeida Lima vor. Wie bereits erwähnt, nahm Moniz nicht auf die frühen psychochirurgischen Eingriffe Burckhardts Bezug.

Mit Berufung auf die Monizschen Operationen führten die Neurologen Walter Freeman und James Watts nur wenig später die ersten Leukotomien in den USA durch ${ }^{141}$. Sie publizierten ihre Arbeiten im angloamerikanischen Sprachraum und sorgten so binnen weniger Jahre für eine weltweite Verbreitung der Methode. Es folgten zahlreiche Modifikationen der Operationstechnik (Topektomie, transorbitale Leukotomie, orbitales Undercutting u.v.m.) und der Zielpunkte im Gehirn (Hypothalamotomie, Cingulotomie, Thalamotomie, Capsulotomie u.s.w. $)^{142}$. Freeman und Watts gehörten zu den wenigen Nervenärzten ihrer Zeit, die Burckhardts Eingriffe erwähnten.

137 Wladimir M. von Bechterew (1857-1927). Nach Bechterew ist die Spondylitis ankylosans benannt. Vgl. auch Morton's Medical Bibliography (oben Anm. 36) 583.

138 Ludwig M. Puusepp (1875-1942). Puusepp wurde in den Zwanzigerjahren durch seine Methode der operativen Entfernung von Hirntumoren bekannt: vgl. Morton's Medical Bibliography (1991) 753.

139 Wladimir M. von Bechterew/Ludwig M. Puusepp, «Surgery in mental diseases», Obozrenie Psychiartrii, Neurologii i Experimentalnoy Psychologii 4 (1908) 208-227 - zitiert nach B. I. Lichtermann, «On the History of Psychosurgery in Russia», Acta Neurochirurgica 125 (1993) 1-4, hier 4.

140 Puusepp beliess es damals bei drei Patienten. Vgl. auch ders., «Alcune considerazioni sugli interventi chirurgici nelle malatie mentali», Giornale della Accademia di Medicina di Torino 100 (1937) 3-16.

141 Walter Freeman/James W. Watts, «Prefrontal lobotomy in agitated depression», Medical Annals of the District of Columbia 5 (1936) 326-328.

142 Für Details vgl. Adler/Saupe, a. O. (oben Anm. 1) sowie Jürgen Hill, Der frontale Eingriff in das Gehirn und die Entwicklung der Psychochirurgie: eine symptomatische Betrachtung der Psychochirurgie, Münster 1992 (= Spuren der Wirklichkeit. Soziologische Beiträge, 1). Seit dem Ende der Siebzigerjahre ging die Zahl der psychochirurgischen Massnahmen im 
In ihrer international bekannten Monographie mit dem Titel Psychochirurgie (1942) stellten beide fest: «His operations can hardly been considered more than mutilating even though they caused surprisingly little aphasia.» ${ }^{143}$

Auch am Ende dieses Jahrhunderts beschäftigt sich die Mehrzahl der Arbeiten zur Geschichte der Psychochirurgie mit der «Ära» Egas Moniz bzw. Walter Freeman ${ }^{144}$. Sofern Gottlieb Burckhardts Topektomien erwähnt werden, geschieht dies meist am Rande oder im Sinne einer kurzen Überleitung $\mathrm{zu}$ «moderneren» psychochirurgischen Verfahren.

Auch hinsichtlich der Bewertung seiner frühen Eingriffe und ihrer ethischen Implikationen herrscht keine Einigkeit. Vielmehr mischten bzw. mischen sich vorsichtige Kommentare mit anerkennenden Stimmen:

In Zurückhaltung üben sich zum Beispiel Adler und Saupe (1979) in ihrem ansonsten sehr detailfreudigen Buch:

«Burckhardts Ideen gerieten wieder in Vergessenheit, weil zu seiner Zeit viele Voraussetzungen für eine Rezeption und Weiterführung des mehr spekulativen Vorgehens fehlten.» ${ }^{145}$

Vorsichtige Kritik melden Joanette et al. (1993) an:

«In some respects, what this young psychiatrist did can be perceived as offensive, despite the fact that his clinical observations remain of interest. Perhaps the most fitting evaluation is that Burckhardt went from theory to practice, in his mind for the benefit of a few unfortunate patients.» ${ }^{146}$

Ein positives Bild des schweizerischen Psychiaters zeichnet Carl Henschen (1955). Er sieht in Burckhardt «eine für damals einzigartige Synthese von Psychiater und Chirurg», der seiner Zeit voraus war:

«In sehr gescheiten, den hirnanatomischen und hirnphysiologischen Erkenntnissen seiner Zeit vorauseilenden Ueberlegungen forderte Burckhardt das therapeutische Recht, «solche Rindenteile auszuschneiden, die man als Ausgangs- und Knotenpunkt psychischer Störungen ansehen kann> ...» ${ }^{147}$

deutschsprachigen Raum wie auch im internationalen Massstab drastisch zurück. Gleichwohl stellte der amerikanische Psychobiologe Elliot S. Valenstein noch 1990 fest: «Psychosurgery is still being performed in many countries, despite the enactment of some legislation restricting its use. The procedures used and the brain targets selected are almost identical to what they were at the end of the 1970s»: ders., a. O. (oben Anm. 12) 550. Eine umfassende Studie zur Entwicklung der Psychochirurgie seit dem Ende des Zweiten Weltkrieges ist derzeit in Vorbereitung.

143 Freeman/Watts, a. O. (oben Anm. 15) 9.

144 Vgl. exemplarisch de Barahona Fernandez, a. O. (oben Anm. 5); Tippin/Henn, a. O. (oben Anm. 5); Almeida Lima, «Egas Moniz», Journal of Neurology 207 (1974) 167-170; Anastasia Kurcharski, «History of Frontal Lobotomy in the United States, 1935-1955», Neurosurgery 14 (1984) 765-772; Victor W. Swayze II, «Frontal Leukotomy and Related Psychosurgical Procedures in the Era Before Antipsychotics (1935-1954): A Historical Overview», American Journal of Psychiatry 152 (1995) 505-515.

145 Adler/Saupe, a. O. (oben Anm. 1) 38.

146 Joanette et al., a. O. (oben Anm. 2) 586.

147 Carl Henschen, «Paralipomena zur Lobotomiefrage», Medizinische Klinik Nr. 32 (1955) 1343-1346, hier 1344. 
Henschen würdigt die «grossen Grundkonzeptionen von G. Burckhardt und E. Moniz» und mahnt das «prophetische Wort G. Burckhardts» an, wonach die ärztliche Pflicht zu helfen zu besserem Wissen antreibe ${ }^{148}$.

Auch Christian Müller (1960) findet anerkennende Worte für das Werk Gottlieb Burckhardts:

«Burckhardt's name will always occupy a place of honour in the history of psychiatry ... because he was a man who did not look at science as a form of recreation based on abstract theories, but as a necessary preparation for therapeutic action..${ }^{149}$

Eine intensive Auseinandersetzung mit Gottlieb Burckhardt ist weiterhin als Desiderat anzusehen. Besonders deutlich wird dies am Beispiel von [Garrison and] Morton's Medical Bibliography: Obwohl jenes 1943 erstmals veröffentlichte Nachschlagewerk mittlerweile bereits in der 5. Auflage (1991) erschienen ist, findet sich zur Person Gottlieb Burckhardts nach wie vor die gleiche, ebenso undifferenzierte wie sachlich falsche Feststellung:

«Burckhardt ... performed frontal lobotomy on four patients in 1890 , with good results in some cases.» ${ }^{150}$

Fest steht indessen, dass Burckhardt in therapeutischer Hinsicht eine grössere Risikobereitschaft zeigte als alle seine zeitgenössischen Kollegen. 1891 charakterisierte er sich selbst folgendermassen:

«Die Naturen der Aerzte sind verschieden. Der eine hält am Grundsatze fest: Primum non nocere. Der andere sagt: melius anceps remedium quam nullum. Ich gehöre allerdings eher der zweiten Categorie an.» ${ }^{151}$

Dass Gottlieb Burckhardt mit dieser Selbsteinschätzung richtig lag, hatte er drei Jahre zuvor mit der Durchführung des ersten psychochirurgischen Eingriffes eindrucksvoll unter Beweis gestellt.

148 Henschen, a. O. (oben Anm. 147) 1346.

149 Müller, «Father of Topectomy» (oben Anm. 2) 463.

150 Wie bereits erwähnt, begann Burckhardt nicht 1890, sondern bereits 1888 mit seinen psychochirurgischen Eingriffen. Auch sind nicht 4, sondern 6 Fallbeispiele überliefert. Schliesslich sind auch die guten Resultate der betreffenden Operationen - wie oben ausgeführt - mit einem Fragezeichen zu versehen: siehe Morton's Medical Bibliography (oben Anm. 36) 749. Vgl. jenes Zitat mit Leslie T[homas] Morton (Hrsg.), [Fielding Hudson] Garrison and [Leslie Thomas] Morton's Medical Bibliography. An Annotated Check-List of Texts Illustrating the History of Medicine, London ${ }^{2} 1954,425$.

151 Burckhardt, a. O. (oben Anm. 9) 547. 